\title{
Rescue of Two Recombinant Canine Distemper Viruses That Separately Express Dabie Bandavirus Gn and $\mathrm{Gc}$ in Vitro
}

Fuxiao Liu ( $D$ laudawn@126.com )

Qingdao Agricultural University https://orcid.org/0000-0002-3078-0613

Jiahui Lin

Qingdao Agricultural University

Qianqian Wang

Qingdao Agricultural University

Hu Shan

Qingdao Agricultural University

\section{Short Report}

Keywords: Severe fever with thrombocytopenia syndrome, Dabie bandavirus, Canine distemper virus, Gn and Gc, Recombinant virus, Vaccine candidate

Posted Date: June 22nd, 2021

DOl: https://doi.org/10.21203/rs.3.rs-634109/v1

License: (9) This work is licensed under a Creative Commons Attribution 4.0 International License. Read Full License 


\section{Abstract}

Severe fever with thrombocytopenia syndrome (SFTS) is an emerging tick-borne zoonosis with a high mortality rate in humans. Additionally, dogs are frequently reported to be infected with this disease. There has been no commercially available vaccine for humans and animals as yet. The SFTS is caused by Dabie bandavirus (DBV), formerly known as SFTS virus. The DBV is now classified into the genus Bandavirus in the family Phenuiviridae. DBV Gn and Gc can induce specific immune responses in vivo. In this study, we used reverse genetics to construct two recombinant canine distemper viruses (rCDV), rCDV$\mathrm{Gn}$ and -Gc, which could express Dabie bandavirus $\mathrm{Gn}$ and $\mathrm{Gc}$ in vitro, respectively. Two foreign sequences, $\mathrm{Gn}$ and $\mathrm{Gc}$ open reading frames, were genetically stable during twenty serial viral passages in cells. Growth curve of the rCDV-Gc basically coincided with that of a wild-type CDV, but showed a significant difference from that of the rCDV-Gn. The rCDV-Gn and -Gc were derived from a common parental CDV, the virulence-attenuating QN strain. Therefore, if proven to be efficient in resisting both canine distemper and SFTS in dogs, either or both recombinant CDVs would be potential vaccine candidates.

\section{Introduction}

Severe fever with thrombocytopenia syndrome (SFTS) was an emerging infectious disease, caused by Dabie bandavirus (DBV), formerly known as SFTS virus. This disease was initially reported in Central China in 2009. Its clinical signs included fever, thrombocytopenia, gastrointestinal symptoms and leukocytopenia in patients. There was an unusually high initial case fatality rate of $30 \%$ (Yu et al. 2011). In recent years, this disease raised serious public health concerns, especially in China (Miao et al. 2020). The SFTS is a tick-borne zoonosis. The DBV can rapidly evolve by gene mutation, reassortment and homologous recombination in ticks and reservoir hosts (Liu et al. 2014). More recently, it was frequently reported that non-human animals, especially dogs, were infected by DBV, or were diagnosed with DBV antibody-positive (Kang et al. 2019; Lee et al. 2017; Lee et al. 2019; Nam et al. 2020; Niu et al. 2013; Tian et al. 2017). Dog-to-human transmission of DBV can even occur through manual de-ticking of domestic dogs (Chung et al. 2020). No specific treatment of SFTS is available now. Development of veterinary vaccines would be one of the most effective ways to protect companion dogs from SFTS, thereby interrupting a potential route of dog-to-human transmission.

The DBV belongs to the genus Bandavirus in the family Phenuiviridae of the order Bunyavirales. Its genome is segmented into three pieces: $L, M$ and $S$ segments. The $M$ segment encodes a membrane protein precursor (Fig. 1A) that matures into two glycoproteins, $\mathrm{Gn}$ and $\mathrm{Gc}$, embedded within the viral envelope (Fig. 1B). Bunyaviral $\mathrm{Gn}$ and $\mathrm{Gc}$ can induce specific immune responses in vivo (Duehr et al. 2020; Said et al. 2017; Wernike et al. 2017; Wright et al. 2020). Different virus-vectored vaccines were reported to be capable of inducing DBV-specific immune responses (Dong et al. 2019; Tian et al. 2021; Yoshikawa et al. 2021; Zhao et al. 2020b). For example, Dong et al (2019) constructed a live-attenuated recombinant vesicular stomatitis virus that could express the DBV Gn/Gc glycoproteins. Single-dose vaccination with it was demonstrated to be able to elicit complete protection in mice against DBV (Dong 
et al. 2019). More recently, Tian et al (2021) reported that Gn-expressing recombinant rabies virus conferred protective immune responses in mice (Tian et al. 2021).

Unfortunately, there has been no report concerning canine distemper virus (CDV)-vectored vaccine against DBV as yet. Canine distemper virus (CDV) causes a highly contagious disease, canine distemper, which affects a wide variety of domestic and wild carnivores (McCarthy et al. 2007). This virus is classified into the genus Morbillivirus in the family Paramyxoviridae. Virulence-attenuating CDV strains were potential vectors for delivering foreign antigens to induce protective immunities against canine distemper and other diseases (Zhao et al. 2020a), such as leishmaniasis (Miura et al. 2015) and rabies (Wang et al. 2012). We have developed one virulence-attenuating CDV (QN strain) previously. Considering anti-DBV vaccines unavailable for dogs, we rescued two recombinant CDVs (QN strain) in this study. These two recombinant CDVs were demonstrated to able of separately expressing DBV Gn and Gc. If proven to elicit protective immunities against canine distemper and SFTS, both viruses would be potential vaccine candidates for dogs.

\section{Materials And Methods}

\section{Cells, virus and plasmids}

The BSR-T7/5 (Buchholz et al. 1999) and Vero-Dog-SLAM (VDS) cells, kindly provided by the China Animal Health and Epidemiology Center, were cultured at $37^{\circ} \mathrm{C}$ with $5 \% \mathrm{CO}_{2}$ in Dulbecco's modified Eagle's medium (DMEM) supplemented with $10 \%$ fetal bovine serum, and containing penicillin (100 $\mathrm{U} / \mathrm{mL})$, streptomycin $(100 \mu \mathrm{g} / \mathrm{mL})$, amphotericin B $(0.25 \mu \mathrm{g} / \mathrm{mL})$ and G418 $(500 \mu \mathrm{g} / \mathrm{mL})$. The wild-type CDV (wt-CDV), QN strain, was propagated in VDS cells. The QN strain-based platform of reverse genetics had been established previously in our laboratory, mainly containing four plasmids, namely one fulllength cDNA clone of recombinant CDV that expressed a foreign protein, and three helper plasmids (pCAGGS-N, pCAGGS-P and pCAGGS-L).

\section{Construction of rCDV-Gn and -Gc cDNA clones}

The rCDV-Gn and -Gc cDNA clones were schematically shown in Fig. 1C and D, respectively. They were separately flanked by the T7 promoter and a hepatitis delta virus ribozyme-T7 terminator fusion sequence at their $5^{\prime}$ and $3^{\prime}$ ends, respectively. In order to optimize protein expression, $\mathrm{Gn}$ and Gc open reading frames (ORFs) (Genbank access No.: MT236316) were subjected to codon optimization based on dogs, and then independently subcloned into the Not l/Pme I sites of another CDV cDNA clone that was constructed previously in our laboratory. Two recombinant plasmids of cDNA clones were separately purified using the PureLink ${ }^{\mathrm{TM}}$ HiPure Plasmid Maxiprep Kit (Thermo Fischer, Carlsbad, USA) according to the manufacturer's instruction.

\section{Rescue and passaging of rCDV-Gn and -Gc}


BSR-T7/5 cells were seeded into a 12 -well plate, and cultured at $37^{\circ} \mathrm{C}$ with $5 \% \mathrm{CO}_{2}$. To rescue recombinant viruses, a cell monolayer at $70 \%$ confluency was co-transfected with either of the cDNA clones $(2.0 \mu \mathrm{g} /$ well), pCAGGS-N (1.0 $\mu \mathrm{g} /$ well), pCAGGS-P $(0.5 \mu \mathrm{g} /$ well $)$ and pCAGGS-L $(0.5 \mu \mathrm{g} /$ well $)$ using Lipofectamine 2000 (Thermo Fisher, Carlsbad, USA) according to the manufacturer's instruction. Two cotransfected cell monolayers were digested with trypsin at $72 \mathrm{~h}$ post transfection (hpt), and then separately co-cultivated with VDS cells in two T25 flasks. The rescued viruses were subjected to serial blind passages in VDS cells.

\section{RT-PCR analysis}

The rCDV-Gn and-Gc were harvested at passage-10 (P10) for extraction of viral RNAs, which were separately used as templates for RT-PCR analysis using the PrimeScript ${ }^{\text {TM }}$ High Fidelity One Step RT-PCR Kit (Takara, Dalian, China). The forward primer (5'-TCAAGAGTATTACTCATGCTTAA-3') targeted the downstream region of P ORF, and the reverse primer (5'-TCGAAGTCGTACACCTCAGTCAT-3') targeted the upstream region of M ORF. The RT-PCR reaction underwent $45^{\circ} \mathrm{C}$ for $10 \mathrm{~min}, 94^{\circ} \mathrm{C}$ for $2 \mathrm{~min}$ and then 30 cycles at $98^{\circ} \mathrm{C}(10 \mathrm{~s}), 55^{\circ} \mathrm{C}(15 \mathrm{~s})$ and $68^{\circ} \mathrm{C}(20 \mathrm{~s})$. The extracted RNAs were simultaneously subjected to PCR analysis using the same primers. The PCR reaction contained $2 \times$ PrimeSTAR Max Premix (Takara, Dalian, China) and underwent 30 cycles at $98^{\circ} \mathrm{C}(10 \mathrm{~s}), 55^{\circ} \mathrm{C}(10 \mathrm{~s})$ and $72{ }^{\circ} \mathrm{C}(10 \mathrm{~s})$. RT-PCR and PCR products were detected by agarose gel electrophoresis, followed by Sanger sequencing for analyzing two RT-PCR products.

\section{Indirect immunofluorescence assay (IFA)}

Recoveries of rCDV-Gn and-Gc were confirmed by IFA. Briefly, two VDS cell monolayers were independently infected with the P15 rCDV-Gn and -Gc for $24 \mathrm{~h}$, and then fixed in $4 \%$ paraformaldehyde at room temperature for $30 \mathrm{~min}$. After fixation, cells were washed four times with PBS, and then permeated with $0.4 \%$ Triton X-100 at room temperature for $30 \mathrm{~min}$. After permeation, cells were washed three times with PBS and blocked in blocking solution at $37^{\circ} \mathrm{C}$ for $1 \mathrm{~h}$. Subsequently, cells were incubated with the anti-CDV monoclonal antibody (MAb, 1: 400 in blocking solution) at $37^{\circ} \mathrm{C}$ for $2 \mathrm{~h}$. After incubation with the primary antibody, cells were washed three times with PBS and incubated with the Alexa Fluor ${ }^{\circledR} 555$ conjugate (Thermo Fisher, Waltham, MA, USA) (1: 250 in blocking solution) at $37^{\circ} \mathrm{C}$ for $1 \mathrm{~h}$. Cells were washed three times with PBS, coated with $90 \%$ glycerin, and visualized under the fluorescence microscope. As a control, one non-infected cell monolayer was subjected to the same treatments.

\section{Mass spectrometry}

The expressions of Gn and Gc were separately analyzed by mass spectrometry (MS) at the Shanghai Bioprofile Biotechnology Co., Ltd (Shanghai, China), as described previously (Liu et al. 2021). In brief, culture supernatants of $\mathrm{P} 10$ progenies were inactivated by $0.1 \%$ formalin at $4{ }^{\circ} \mathrm{C}$ for $48 \mathrm{~h}$. Protein digestion was performed with a method of filter-aided sample preparation (Wiśniewski et al. 2009). Liquid chromatography linked to tandem mass spectrometry (LC-MS/MS) was performed on a Q Exactive Plus mass spectrometer that was coupled to Easy nLC (Thermo Fisher, Waltham, MA, USA). The MS data 
were analyzed using MaxQuant software v1.6.0.16. The database search results were filtered and exported with $<1 \%$ false discovery rate at peptide-spectrum-matched level, and protein level, respectively.

\section{Growth kinetics}

The rCDV-Gn and -Gc were compared with each other on their growth kinetics in vitro. Briefly, VDS cells were seeded into five 12-well plates ( $10^{6}$ cells/well, and 6 wells/plate) for incubation at $37^{\circ} \mathrm{C}$ for $2 \mathrm{~h}$. The P15 rCDV-Gn and -Gc were separately inoculated $(\mathrm{MOI}=0.0002)$ into all plates (3 wells/progeny in each plate) for incubation at $37^{\circ} \mathrm{C}$ for $3 \mathrm{~h}$, and then supernatants were replaced with DMEM for further incubation at $37^{\circ} \mathrm{C}$. At $0,24,48,72$ and $96 \mathrm{~h}$ post inoculation (hpi), any of plates was randomly removed from the incubator, and subjected to two freeze-and-thaw cycles to collect supernatant for viral titration using the Spearman-Kärber equation (Finney 1952). The wt-CDV as a control was subjected to the same treatments. Kinetic curve of virus growth was drawn using the GraphPad Prism software (Version 7.0). Data at each time point were representative of three independent experiments.

\section{Genetic stabilities of two foreign sequences}

Two recombinant viruses were subjected to twenty serial passages ( $3 \mathrm{~d} /$ passage) in VDS cells. Their culture supernatants at P15 and P20 were harvested for RT-PCR analysis, as described in Subheading RTPCR analysis. Two RT-PCR products were detected by agarose gel electrophoresis, followed by Sanger sequencing.

\section{Results}

\section{Rescue of rCDV-Gn and -Gc}

The co-transfection of four plasmids was carried out in BSR-T7/5 cells that could express the T7 RNA polymerase. Owing to the absence of CDV receptors on it, the BSR-T7/5 cell line was only used for virus recovery, rather than passaging. Alternatively, the VDS cell line, because permissive to CDV infection, was used for serial blind passages of rescued viruses in this study. Typical cytopathic effects (CPEs), such as exacerbated cell-to-cell fusion (Fig. 2A) and syncytium formation (Fig. 2B), appeared on VDS cell monolayers with viral passaging. The CPEs were also visible during serial blind passages.

\section{RT-PCR analysis of rCDV-Gn and -Gc}

The P10 rCDV-Gn and -Gc were simultaneously analyzed by RT-PCR to confirm their identities. Two expected bands, 1887 (Fig. 2C) and 1899 bps (Fig. 2D), were observable only on the RT-PCR lanes by agarose gel electrophoresis. As a control, PCR analysis (Fig. 2C and D, Lane PCR) showed no residue of cDNA clone plasmid affecting the RT-PCR analysis. The identities of rCDV-Gn and -Gc were confirmed by Sanger sequencing.

\section{IFA and mass spectrometry}


In order to confirm recovery of rCDV-Gn and -Gc, the IFA was performed using the anti-CDV MAb as the primary antibody. Bright red syncytia were visible on the rCDV-Gn- and rCDV-Gc-infected cell monolayers. As a control, non-infected cells did not show a similar phenotype (Fig. 2E). The IFA result confirmed that two competent recombinant CDVs had been recovered from their cDNA clones. Expressions of $\mathrm{G}$ and Gc were demonstrated by mass spectrometry, which exhibited that $\mathrm{Gn}$ - and Gc-specific peptide sequences matched to the MS/MS spectra. Two representative MS/MS spectra were shown in Fig. 3A and B.

\section{Growth kinetics of rCDV-Gn and -Gc}

To determine growth curves of both viruses in vitro, VDS cell monolayers were independently inoculated with the P15 rCDV-Gn and -Gc. Typical syncytia were visible at $24 \mathrm{hpi}$, and exacerbated over time to cause intercellular hyperfusogenicity at $48 \mathrm{hpi}$ (Fig. $3 \mathrm{C}$ and D). The growth curves of both viruses were compared with each other and with that of the wt-CDV (Fig. 3E). These two recombinants exhibited different growth kinetics: the rCDV-Gn replicated more slowly during 0 and $24 \mathrm{hpi}$, but maintained a higher titer level than the rCDV-Gc did during 48 to $96 \mathrm{hpi}$. The rCDV-Gc showed the similar growth kinetics to that of the wt-CDV.

\section{Genetic stability of foreign sequences}

To test genetic stability of two foreign sequences, both the rCDV-Gn and -Gc were serially passaged in VDS cells for a total of twenty passages. The RT-PCR showed that individual specific products were separately amplified from RNA samples of P15 and P20 progenies (Fig. 3F). The P20 RT-PCR products were collected for Sanger sequencing, indicating no point mutation appearing in the foreign sequence of rCDV-Gn. The rCDV-Gc showed a nonsynonymous mutation (A157C) in the Gc ORF, correspondingly resulted in a mutation of amino acid (T53P) in Gc.

\section{Discussion}

In recent years, the SFTS was frequently reported in China, Japan, and the Republic of Korea. This disease, characterized by a high case-fatality rate in humans, is primarily transmitted via tick bite, and can also be transmitted from person to person through contacting patient's blood (Liu et al. 2012). Domesticated animals, like companion dogs, should be considered as a source of animal-to-human transmission, as evidenced by recent case reports (Chung et al. 2020; Han et al. 2020; Kang et al. 2019; Nam et al. 2020). Unfortunately, there has been no commercially available vaccine against SFTS for dogs as yet. CDVs are efficient vectors for expressing heterologous proteins (Liu et al. 2020; Ludlow et al. 2012; Parks et al. 2002; Plattet et al. 2004), or antigens that can confer specific immune responses in animals (Li et al. 2015; Miura et al. 2015; Wang et al. 2012). This prompted us to develop new generation CDV vaccines using reverse genetics for delivering DBV antigens to induce protective immunity in dogs.

We have recently constructed a platform of CDV (5804P strain) reverse genetics for rescue of recombinant virus (Liu et al. 2020). In the present study, we rescued two recombinant virulenceattenuating CDVs (QN strain), independently coding for DBV Gn and Gc in cells. The reason why the DBV glycoprotein precursor (Gn-Gc) was not used for construction of recombinant CDV was that the full-length 
sequence of Gn-Gc was theoretically too long (3222 nt) to be accommodated in a CDV genome. Even if a Gn-Gc-inserting CDV can be rescued from its recombinant cDNA clone, both viral replication and protein expression would be affected by the excessive load of heterologous sequence in a CDV genome to some extent. Therefore, we independently rescued $\mathrm{Gn}$ - and Gc-expressing CDVs, in order to maintain the viral propagation that was not significantly affected by foreign sequences.

In initial blind passages after co-transfection, both recombinants revealed a weak adaptability in VDS cells, as evidenced by slow appearance of virus-induced CPE foci (data not shown). Such a weak adaptability was gradually improved with serial passaging in VDS cells. Each viral progeny is theoretically better than its previous one in growth kinetics during initial blind passages (Liu et al. 2016). We speculated that both recombinants had been basically adapted to the VDS cell line at P15. Thus, the P15 progenies were used for determining the growth curves of two recombinant viruses. The rCDV-Gc had a similar growth curve to that of the wt-CDV. The rCDV-Gn showed totally different growth kinetics from those of the rCDV-Gc and wt-CDV, implying that the Gn sequence exerted an impact on viral replication in vitro. Nevertheless, Tian et al (2021) recently revealed that the insertion of DBV Gn did not affect replication of a recombinant rabies virus in vitro, compared with that of its parental strain (Tian et al. 2021). We recently rescued another recombinant CDV QN strain that expressed a SARS-CoV-2 S1 subunit (686 aa) in VDS cells. The rCDV-Gn was measured to have a similar growth curve to that of the S1 subunit-expressing CDV (data not shown).

To enhance expression levels of $\mathrm{Gn}$ and $\mathrm{Gc}$, their full-length ORFs were subjected to codon optimization based on dogs. Owing to neither Gn- nor Gc-specific antibody available in our laboratory, their expressions were qualitatively analyzed by mass spectrometry, demonstrating that the rCDV-Gn and -Gc were able of encoding the Gn and Gc in VDS cells, respectively. Unfortunately, the protein expression was not quantitatively analyzed by Western blot or other methods, due to absence of Gn- and Gc-specific antibodies. Because virus-vectored vaccines are actually live recombinant viruses, over-expression of foreign antigens is not always advantageous for vaccines to elicit protective immunity in animals. A given foreign antigen should be expressed at a proper, but not high, level in a host (Liu et al. 2019). Our present study revealed that both recombinant CDVs could independently express $\mathrm{Gn}$ and Gc in vitro. If demonstrated to be efficient against both canine distemper and SFTS in dogs in future, either or both recombinants would be potential vaccine candidates.

\section{Declarations}

\section{Acknowledgements}

We thank other members in our group for their assistance in cell culture and virus passaging.

\section{Authors' contributions}

FL conducted experiments and wrote the manuscript. JL and QW performed the experimental works. HS provided the funding, and supervised the project. 


\section{Funding}

This work was supported by the Shandong Key Research and Development Program, China (2019GNC106074), and the Research Foundation for Distinguished Scholars of Qingdao Agricultural University (1120045), China.

\section{Data availability}

All data generated or analyzed during this study are included in this published article.

\section{Ethics approval}

Not applicable.

\section{Consent to participate and consent for publication}

All authors agreed to participate in this work. They read and approved the final manuscript.

\section{Conflict of interest}

The authors declare no conflict of interest.

\section{Disclosure statement}

No competing financial interest exists.

\section{References}

1. Buchholz UJ, Finke S, Conzelmann KK (1999) Generation of bovine respiratory syncytial virus (BRSV) from cDNA: BRSV NS2 is not essential for virus replication in tissue culture, and the human RSV leader region acts as a functional BRSV genome promoter. J Virol 73:251-259

2. Chung JK, Kim CM, Kim DM, Yun NR, Park JW, Seo J, Kim YS (2020) Severe Fever with Thrombocytopenia Syndrome Associated with Manual De-Ticking of Domestic Dogs. Vector Borne Zoonotic Dis 20:285-294

3. Dong F et al. (2019) Single dose of a rVSV-based vaccine elicits complete protection against severe fever with thrombocytopenia syndrome virus. NPJ Vaccines 4:5

4. Duehr J et al. (2020) Neutralizing Monoclonal Antibodies against the $G n$ and the Gc of the Andes Virus Glycoprotein Spike Complex Protect from Virus Challenge in a Preclinical Hamster Model. mBio 11

5. Finney DJ (1952) Statistical Method in Biological Assay vol first ed. Charles Griffin and Company, London

6. Han SW, Kang JG, Byeon AR, Cho YK, Choi KS, Chae JS (2020) Severe fever with thrombocytopenia syndrome in canines from the Republic of Korea. Ticks Tick Borne Dis 11:101454 
7. Kang JG, Cho YK, Jo YS, Chae JB, Joo YH, Park KW, Chae JS (2019) Severe Fever with Thrombocytopenia Syndrome Virus in Dogs, South Korea. Emerg Infect Dis 25:376-378

8. Lee SH et al. (2017) Molecular detection and phylogenetic analysis of severe fever with thrombocytopenia syndrome virus in shelter dogs and cats in the Republic of Korea. Ticks Tick Borne Dis 8:626-630

9. Lee SY et al. (2019) Complete Genome Sequences of Two Severe Fever with Thrombocytopenia Syndrome Virus Strains Isolated from a Human and a Dog in the Republic Of Korea. Microbiol Resour Announc 8

10. Li Z et al. (2015) A recombinant canine distemper virus expressing a modified rabies virus glycoprotein induces immune responses in mice. Virus Genes 50:434-441

11. Liu F, Huang Y, Wang Q, Li J, Shan H (2021) Rescue of Senecavirus A to uncover mutation profiles of its progenies during 80 serial passages in vitro. Vet Microbiol 253:108969

12. Liu F, Li L, Liu Y, Sun C, Liu C, Wu X, Wang Z (2019) Development of reverse genetics system for small ruminant morbillivirus: Rescuing recombinant virus to express Echinococcus granulosus EG95 antigen. Virus Res 261:50-55

13. Liu F, Wang Q, Huang Y, Wang N, Zhang Y, Shan H (2020) Recovery of NanoLuc Luciferase-Tagged Canine Distemper Virus for Facilitating Rapid Screening of Antivirals in vitro. Front Vet Sci 7:600796

14. Liu F, Wu X, Li L, Zou Y, Liu S, Wang Z (2016) Evolutionary characteristics of morbilliviruses during serial passages in vitro: Gradual attenuation of virus virulence. Comp Immunol Microbiol Infect Dis 47:7-18

15. Liu Q, He B, Huang SY, Wei F, Zhu XQ (2014) Severe fever with thrombocytopenia syndrome, an emerging tick-borne zoonosis. Lancet Infect Dis 14:763-772

16. Liu Y et al. (2012) Person-to-person transmission of severe fever with thrombocytopenia syndrome virus. Vector Borne Zoonotic Dis 12:156-160

17. Ludlow M et al. (2012) Recombinant canine distemper virus strain Snyder Hill expressing green or red fluorescent proteins causes meningoencephalitis in the ferret. J Virol 86:7508-7519

18. McCarthy AJ, Shaw MA, Goodman SJ (2007) Pathogen evolution and disease emergence in carnivores. Proc Biol Sci 274:3165-3174

19. Miao D et al. (2020) Epidemiology and Ecology of Severe Fever with Thrombocytopenia Syndrome in China, 2010-2018. Clin Infect Dis

20. Miura R et al. (2015) Efficacy of Recombinant Canine Distemper Virus Expressing Leishmania Antigen against Leishmania Challenge in Dogs. PLoS Negl Trop Dis 9:e0003914

21. Nam SJ, Oh YI, Kim HJ, Cheon DS, Noh SJ, Hong YJ (2020) Unusual case of severe fever with thrombocytopenia syndrome showing clinical manifestations in a companion dog. Vet Med Sci 6:353-358

22. Niu G et al. (2013) Severe fever with thrombocytopenia syndrome virus among domesticated animals, China. Emerg Infect Dis 19:756-763 
23. Parks CL et al. (2002) Expression of a foreign gene by recombinant canine distemper virus recovered from cloned DNAs. Virus Res 83:131-147

24. Plattet P, Zweifel C, Wiederkehr C, Belloy L, Cherpillod P, Zurbriggen A, Wittek R (2004) Recovery of a persistent Canine distemper virus expressing the enhanced green fluorescent protein from cloned cDNA. Virus Res 101:147-153

25. Said A, Elmanzalawy M, Ma G, Damiani AM, Osterrieder N (2017) An equine herpesvirus type 1 (EHV1) vector expressing Rift Valley fever virus (RVFV) Gn and Gc induces neutralizing antibodies in sheep. Virol J 14:154

26. Tian H et al. (2017) Severe Fever with Thrombocytopenia Syndrome Virus in Humans, Domesticated Animals, Ticks, and Mosquitoes, Shaanxi Province, China. Am J Trop Med Hyg 96:1346-1349

27. Tian $L$ et al. (2021) A rabies virus vectored severe fever with thrombocytopenia syndrome (SFTS) bivalent candidate vaccine confers protective immune responses in mice. Vet Microbiol 257:109076

28. Wang $X$ et al. (2012) Recombinant canine distemper virus serves as bivalent live vaccine against rabies and canine distemper. Vaccine 30:5067-5072

29. Wernike K, Aebischer A, Roman-Sosa G, Beer M (2017) The N-terminal domain of Schmallenberg virus envelope protein $\mathrm{Gc}$ is highly immunogenic and can provide protection from infection. Sci Rep 7:42500

30. Wiśniewski JR, Zougman A, Nagaraj N, Mann M (2009) Universal sample preparation method for proteome analysis. Nat Methods 6:359-362

31. Wright D et al. (2020) Naturally Acquired Rift Valley Fever Virus Neutralizing Antibodies Predominantly Target the Gn Glycoprotein. iScience 23:101669

32. Yoshikawa T et al. (2021) A highly attenuated vaccinia virus strain LC16m8-based vaccine for severe fever with thrombocytopenia syndrome. PLoS Path 17:e1008859

33. Yu XJ et al. (2011) Fever with thrombocytopenia associated with a novel bunyavirus in China. New Engl J Med 364:1523-1532

34. Zhao J, Ren Y, Chen J, Zheng J, Sun D (2020a) Viral Pathogenesis, Recombinant Vaccines, and Oncolytic Virotherapy: Applications of the Canine Distemper Virus Reverse Genetics System. Viruses 12:339

35. Zhao Z et al. (2020b) Recombinant Human Adenovirus Type 5 Co-expressing RABV G and SFTSV Gn Induces Protective Immunity Against Rabies Virus and Severe Fever With Thrombocytopenia Syndrome Virus in Mice. Front Microbiol 11:1473

\section{Figures}


(A)

\section{VRIEMVRP}

\begin{tabular}{|l|l|}
\hline Gn (535 aa) & Gc (538 aa) \\
\hline
\end{tabular}

(C)

\begin{tabular}{|l|l|l|l|l|l|l|l|l|}
\hline M GS & G ORF \\
\hline
\end{tabular}

(B)

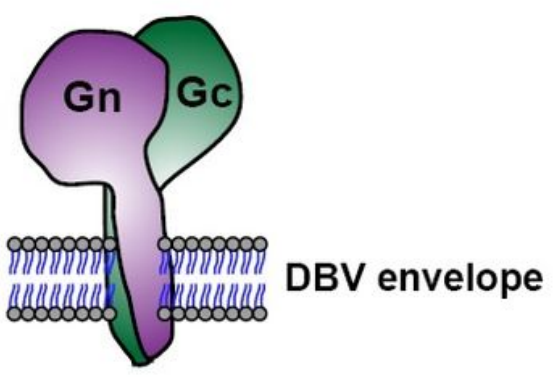

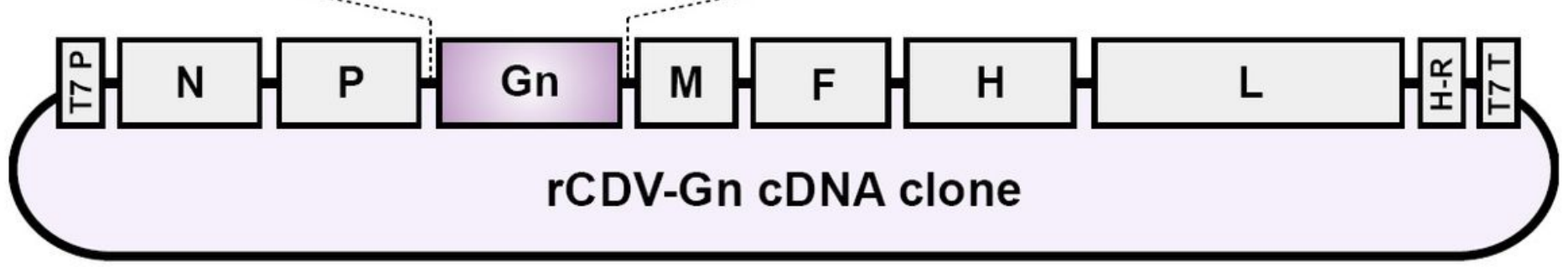

(D)
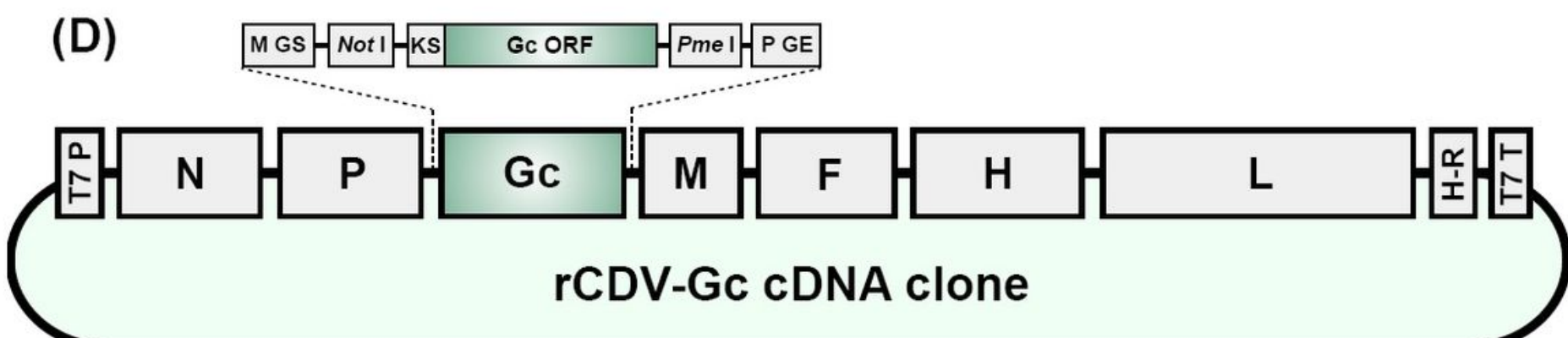

Figure 1

Schematic representations of DBV glycoprotein precursor, Gn-Gc heterodimer, rCDV-Gn cDNA clone and rCDV-Gc cDNA clone. DBV glycoprotein precursor composed of $\mathrm{Gn}$ and $\mathrm{Gc}(\mathrm{A})$. Their cleavage site is marked with red arrow. DBV spike composed of $\mathrm{Gn}-\mathrm{Gc}$ heterodimer that is embedded within viral envelope (B). rCDV-Gn cDNA clone (C) and rCDV-Gc cDNA clone (D). T7 P: T7 promoter; GS: gene start; GE: gene end; KS: Kozak sequence; H-R: hepatitis delta virus ribozyme; T7 T: T7 terminator. 
(A)

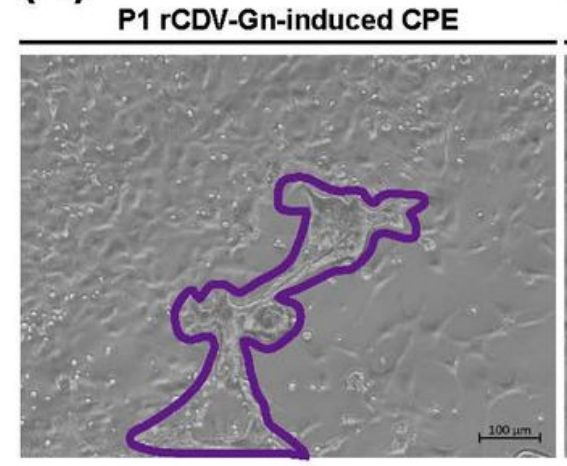

(B)

P2 rCDV-Gc-induced CPE
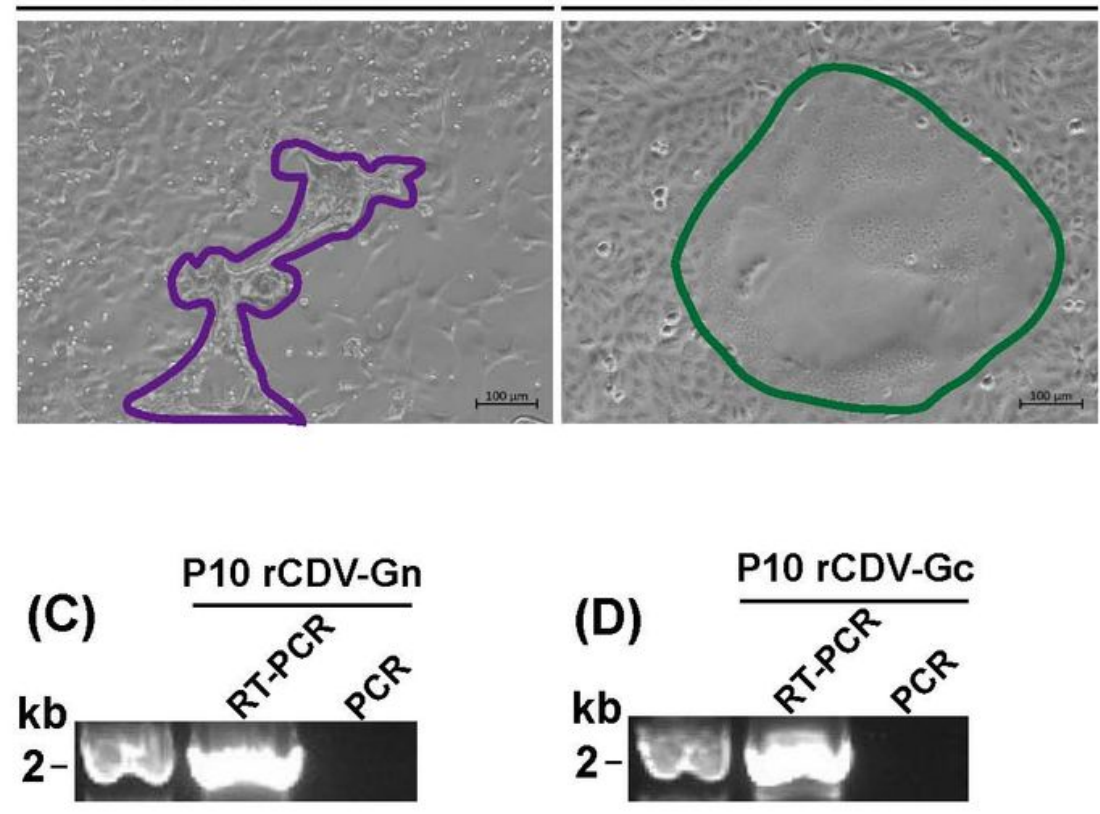

(E)

IFA of rCDV-Gn-infected cells

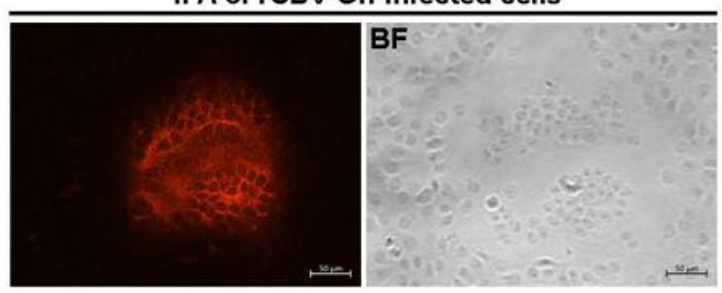

IFA of rCDV-Gc-infected cells

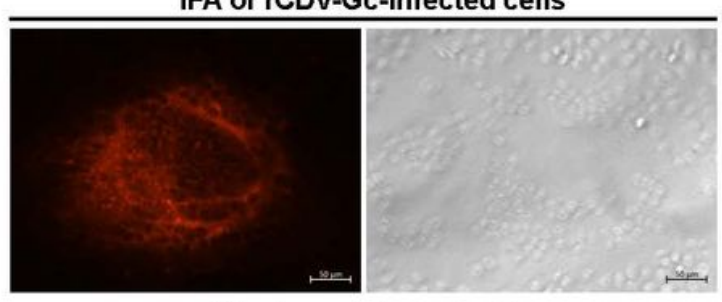

IFA of non-infected cells

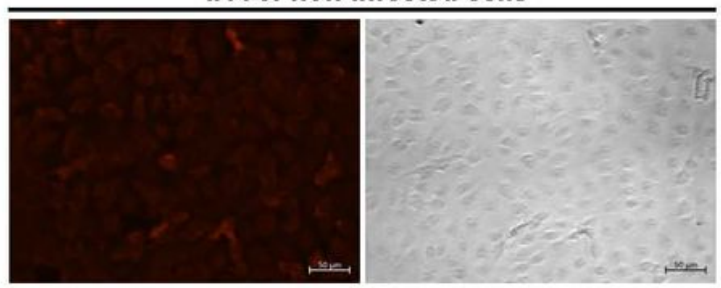

\section{Figure 2}

Rescue and identification of rCDV-Gn and -Gc. rCDV-Gn-induced cell-to-cell fusion (A, enclosed by purple line) and rCDV-Gc-induced syncytium formation (B, enclosed by green line) on VDS cell monolayers during viral passaging. RT-PCR detection of the P10 rCDV-Gn (C) and -Gc (D) using the forward (5'TCAAGAGTATTACTCATGCTTAA-3') and reverse (5'-TCGAAGTCGTACACCTCAGTCAT-3') primers. The Gnand Gc-specific bands are 1887 and 1899 bps, respectively. Indirect immunofluorescence assay of VDS cell monolayers separately inoculated with rCDV-Gn and -Gc $(E)$. The primary and secondary antibodies are anti-CDV MAb and Alexa Fluor ${ }^{\circledR} 555$ antibody, respectively. 
(A)

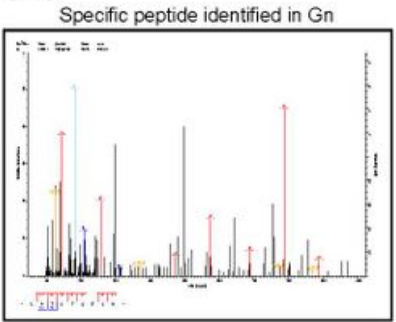

(B)

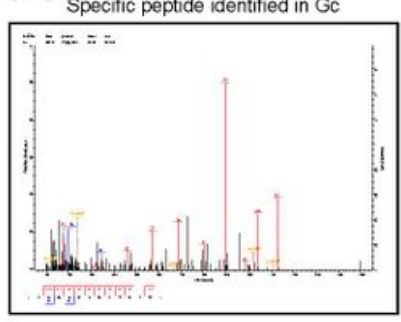

(E)

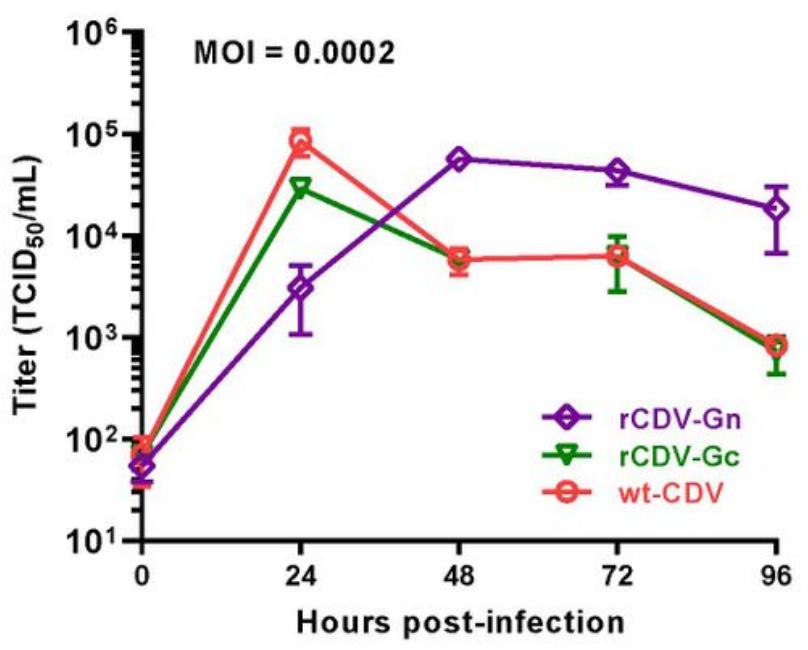

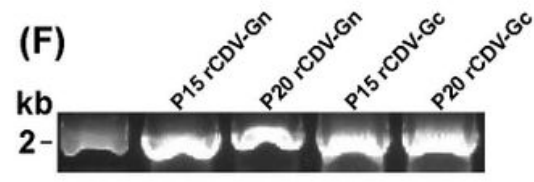

(C)

rCDV-Gn-induced CPE at $0,24,48,72$ and 96 hpi $(\mathrm{MOI}=0.0002)$
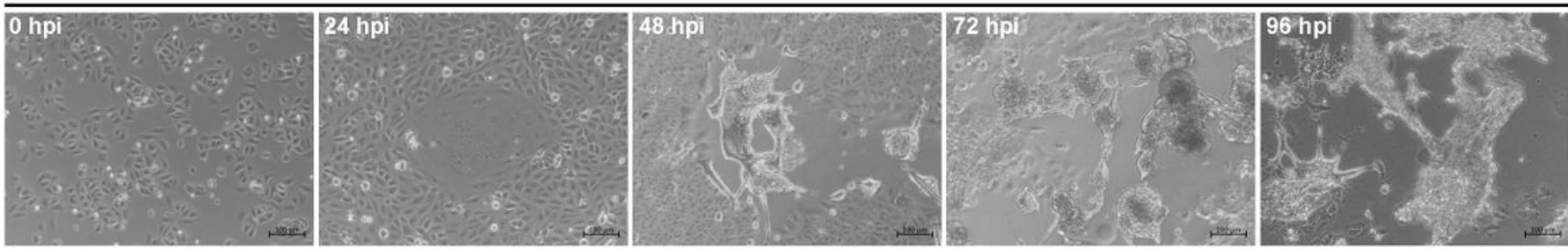

(D)

rCDV-Gc-induced CPE at $0,24,48,72$ and $96 \mathrm{hpi}(\mathrm{MOI}=0.0002)$

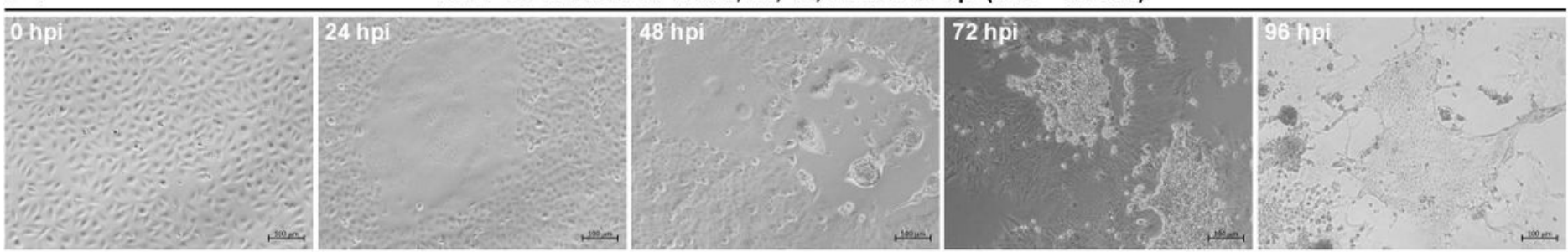

Figure 3

Characterization of rCDV-Gn and -Gc. Representative Gn- (A) and Gc (B)-specific MS/MS spectra on peptide identifications. CPEs on VDS cell monolayers separately inoculated $(\mathrm{MOI}=0.0002)$ with the P15 rCDV-Gn (C) and -Gc (D) at 0, 24, 48, 72 and 96 hpi. Multi-step growth curves of the P15 rCDV-Gn and -Gc (E). Growth curves are drawn using the GraphPad Prism software (Version 7.0). Data at each time point are representative of three independent experiments. RT-PCR analysis on P15 and P20 progenies of rCDVGn and -Gc using the forward (5'-TCAAGAGTATTACTCATGCTTAA-3') and reverse (5'TCGAAGTCGTACACCTCAGTCAT-3') primers (F). 